\title{
HIGHER-ORDER LORENTZ VIOLATIONS IN ELECTRODYNAMICS
}

\author{
Matthew Mewes \\ Department of Physics and Astronomy, Swarthmore College \\ Swarthmore, PA 19081, U.S.A.
}

\begin{abstract}
The Standard-Model Extension (SME) provides a theoretical framework for tests of Lorentz invariance. To date, most studies have focused on the minimal SME, which restricts attention to operators of renormalizable dimension. Here, we review recent studies involving the nonrenormalizable photon sector of the SME.
\end{abstract}

\section{Introduction}

Experiments involving photons have led to some of the most precise tests of Lorentz invariance. ${ }^{1}$ These include tests involving resonant cavities, ${ }^{2}$ cosmic birefringence, ${ }^{3-5}$ and accelerators. ${ }^{6}$ Most searches for Lorentz violation in photons have been analyzed using the minimal version of the StandardModel Extension (SME). ${ }^{7}$ The SME provides the theoretical foundation for Lorentz tests involving any of the particles in the Standard Model of particle physics and gravity. In its simplest form, the minimal StandardModel Extension (mSME) restricts attention to operators that obey the usual spacetime-translational and gauge symmetries and restricts attention to operators of renormalizable dimension, $d=3,4$.

The photon sector of the mSME is given by

$$
\mathcal{L}=-\frac{1}{4} F_{\mu \nu} F^{\mu \nu}+\frac{1}{2}\left(k_{A F}\right)^{\kappa} \epsilon_{\kappa \lambda \mu \nu} A^{\lambda} F^{\mu \nu}-\frac{1}{4}\left(k_{F}\right)_{\kappa \lambda \mu \nu} F^{\kappa \lambda} F^{\mu \nu} .
$$

In addition to the usual Maxwell term, this lagrangian includes two Lorentzviolating terms, one for CPT-violating dimension-3 operators with coefficients $\left(k_{A F}\right)^{\kappa}$ and another for CPT-conserving dimension-4 operators with coefficients $\left(k_{F}\right)_{\kappa \lambda \mu \nu}$. The renormalizable condition greatly restricts the number of Lorentz-violating operators. There are a total of four independent CPT-odd coefficients and nineteen independent CPT-even coefficients. The photon sector of the full SME includes many more terms. Here, we re- 
view a recent study of nonrenormalizable operators in the photon sector of the SME. ${ }^{3}$

\section{Nonrenormalizable coefficients}

Allowing for operators of arbitrary dimension, but restricting attention to those that maintain the usual gauge invariance, we arrive at a theory that is given by the Lagrange density

$$
\mathcal{L}=-\frac{1}{4} F_{\mu \nu} F^{\mu \nu}+\frac{1}{2} \epsilon^{\kappa \lambda \mu \nu} A_{\lambda}\left(\hat{k}_{A F}\right)_{\kappa} F^{\mu \nu}-\frac{1}{4} F_{\kappa \lambda}\left(\hat{k}_{F}\right)^{\kappa \lambda \mu \nu} F_{\mu \nu} .
$$

Here we only consider terms quadratic in the photon field $A_{\mu}$, leading to a linear theory. The above equation is written in a form that resembles the mSME expression (1). The key difference is that here the $\hat{k}_{A F}$ and $\hat{k}_{F}$ are operators that depend on the 4 -momentum $p_{\mu}=i \partial_{\mu}$. The effects of these operators mimic the effects of a permeable medium whose activity depends on the photon energy and momentum. This introduces new frequency and direction dependences that do not arise in the mSME case.

The $\hat{k}_{A F}$ and $\hat{k}_{F}$ operators can be expanded in $\partial_{\mu}$, giving the expressions

$$
\begin{aligned}
\left(\hat{k}_{A F}\right)_{\kappa} & =\sum\left(k_{A F}^{(d)}\right)_{\kappa}{ }^{\alpha_{1} \ldots \alpha_{(d-3)}} \partial_{\alpha_{1}} \ldots \partial_{\alpha_{(d-3)}}, \\
\left(\hat{k}_{F}\right)^{\kappa \lambda \mu \nu} & =\sum\left(k_{F}^{(d)}\right)^{\kappa \lambda \mu \nu \alpha_{1} \ldots \alpha_{(d-4)}} \partial_{\alpha_{1}} \ldots \partial_{\alpha_{(d-4)}},
\end{aligned}
$$

where we sum over the dimension $d$ of the associated operator. The CPTodd coefficients $\left(k_{A F}^{(d)}\right)_{\kappa}{ }^{\alpha_{1} \ldots \alpha_{(d-3)}}$ are nonzero for odd $d \geq 3$. The CPT-even coefficients $\left(k_{F}^{(d)}\right)^{\kappa \lambda \mu \nu \alpha_{1} \ldots \alpha_{(d-4)}}$ are nonzero for even $d \geq 4$. The mSME case corresponds to $d=3,4$. The number of coefficients for a given dimension scales like $d^{3}$ for large $d$.

The large number of $k_{A F}^{(d)}$ and $k_{F}^{(d)}$ coefficients and their relatively complicated symmetries implies that a systematic decomposition into a minimal set of independent components is useful. One decomposition uses tensor spherical harmonics. The idea is that each term in the sums in Eq. (3) takes the form of a polynomial in frequency $\omega=p^{0}$ and momentum $\vec{p}$. We can then expand these terms in spin-weighted spherical harmonics ${ }_{s} Y_{j m}(\hat{p})$. The symmetries of the $k_{A F}^{(d)}$ and $k_{F}^{(d)}$ tensors then impose constraints on the spherical coefficients in the expansion. These constraints can be used to find a set of independent coefficients.

As an illustration, the CPT-odd coefficients split into four sets of spherical coefficients:

$$
k_{A F}^{(d)} \rightarrow k_{(V) j m}^{(d)} \oplus\left(\vec{k}_{A F}^{(d)}\right)_{n j m}^{(0 B)} \oplus\left(\vec{k}_{A F}^{(d)}\right)_{n j m}^{(1 B)} \oplus\left(\vec{k}_{A F}^{(d)}\right)_{n j m}^{(1 E)} .
$$


Table 1. Summary of spherical coefficients for Lorentz violation and their index ranges.

\begin{tabular}{c|c|c|c|c} 
coeff. & $d$ & $n$ & $j$ & number \\
\hline \hline$c_{(I) j m}^{(d)}$ & even, $\geq 4$ & - & $0,1, \ldots, d-2$ & $(d-1)^{2}$ \\
$k_{(E) j m}^{(d)}$ & even, $\geq 4$ & - & $2,3, \ldots, d-2$ & $(d-1)^{2}-4$ \\
$k_{(B)}^{(d)}(B)$ & even, $\geq 4$ & - & $2,3, \ldots, d-2$ & $(d-1)^{2}-4$ \\
$k_{(V) j m}^{(d)}$ & odd, $\geq 3$ & - & $0,1, \ldots, d-2$ & $(d-1)^{2}$ \\
\hline$\left(\vec{c}_{F}^{(d)}\right)_{n j m}^{(0 E)}$ & even, $\geq 4$ & $0, \ldots, d-4$ & $n, n-2, \ldots, \geq 0$ & $\frac{(d-1)(d-2)(d-3)}{6}$ \\
$\left(\vec{k}_{F}^{(d)}\right)_{n j m}^{(0 E)}$ & even, $\geq 6$ & $1, \ldots, d-4$ & $n, n-2, \ldots, \geq 0$ & $\frac{(d-1)(d-2)(d-3)-6}{6}$ \\
$\left(\vec{k}_{F}^{(d)}\right)_{n j m}^{(1 E)}$ & even, $\geq 6$ & $1, \ldots, d-4$ & $n+1, n-1, \ldots, \geq 1$ & $\frac{(d-4)\left(d^{2}+d+3\right)}{6}$ \\
$\left(\vec{k}_{F}^{(d)}\right)_{n j m}^{(2 E)}$ & even, $\geq 6$ & $2, \ldots, d-4$ & $n, n-2, \ldots, \geq 2$ & $\frac{(d-4)\left(d^{2}-2 d-9\right)}{6}$ \\
$\left(\vec{k}_{F}^{(d)}\right)_{n j m}^{(1 B)}$ & even, $\geq 6$ & $1, \ldots, d-4$ & $n, n-2, \ldots, \geq 1$ & $\frac{d(d-2)(d-4)}{6}$ \\
$\left(\vec{k}_{F}^{(d)}\right)_{n j m}^{(2 B)}$ & even, $\geq 6$ & $1, \ldots, d-4$ & $n+1, n-1, \ldots, \geq 2$ & $\frac{(d+3)(d-2)(d-4)}{6}$ \\
$\left.\left(\vec{k}_{A F}^{(d)}\right)_{n j m}^{(0 B}\right)$ & odd, $\geq 5$ & $0, \ldots, d-4$ & $n, n-2, \ldots, \geq 0$ & $\frac{(d-1)(d-2)(d-3)}{6}$ \\
$\left(\vec{k}_{A F}^{(d)}\right)_{n j m}^{(1 B)}$ & odd, $\geq 5$ & $0, \ldots, d-4$ & $n+1, n-1, \ldots, \geq 1$ & $\frac{(d+1)(d-1)(d-3)}{6}$ \\
$\left(\vec{k}_{A F}^{(d)}\right)_{n j m}^{(1 E)}$ & odd, $\geq 5$ & $1, \ldots, d-3$ & $n, n-2, \ldots, \geq 1$ & $\frac{(d+1)(d-1)(d-3)}{6}$
\end{tabular}

The notation is chosen to provide information about the properties of the associated operators. The index $d$ is the dimension of the operator. The $j$ and $m$ are the usual spherical-harmonic indices and determine behavior under rotations. The index $n$ gives information concerning the frequency/wavelength dependence of the operator. The remaining indices, in parentheses, give the helicity and parity of the operator. Parity is labeled as either $E$-type, $(-1)^{j}$, or $B$-type, $(-1)^{j+1}$. Birefringent coefficients are labeled by $k$, while $c$ denotes nonbirefringent coefficients. Finally, the negation symbol $\neg$ is used to indicate vacuum-orthogonal coefficients - those that do not affect light propagating in a vacuum, at leading order. Those that affect light appear without the $\neg$ symbol and are referred to as vacuum coefficients. The sets of spherical coefficients for Lorentz violation for both CPT-odd and CPT-even operators are summarized in Table 1.

\section{Astrophysical tests}

The split between vacuum and vacuum-orthogonal coefficients is convenient because defects in the behavior of light can be constrained with extreme precision using sources at cosmological distances $\sim 10^{42} \mathrm{GeV}^{-1}$. The key equation for these tests is the velocity of light in empty space,

$$
v \simeq 1-\varsigma^{0} \pm \sqrt{\left(\varsigma^{1}\right)^{2}+\left(\varsigma^{2}\right)^{2}+\left(\varsigma^{3}\right)^{2}},
$$


where

$$
\begin{aligned}
\varsigma^{0} & =\sum E^{d-4}(-1)^{j}{ }_{0} Y_{j m}(\hat{\boldsymbol{p}}) c_{(I) j m}^{(d)}, \\
\varsigma^{1} \pm i \varsigma^{2} & =\sum E^{d-4}(-1)^{j}{ }_{ \pm 2} Y_{j m}(\hat{\boldsymbol{p}})\left(k_{(E) j m}^{(d)} \mp i k_{(B) j m}^{(d)}\right), \\
\varsigma^{3} & =\sum E^{d-4}(-1)^{j}{ }_{0} Y_{j m}(\hat{\boldsymbol{p}}) k_{(V) j m}^{(d)} .
\end{aligned}
$$

Several unconventional properties result from these expressions. First, the two signs in Eq. (5) are associated with two different polarizations. This leads to birefringence and an evolution of polarization as light propagates. Extremely tight constraints have been placed on birefringence using light from distant sources, such as GRBs and the CMB. ${ }^{4,5}$ Lorentz violation also leads to direction dependence in the speed of light. All coefficients with $j \neq 0$ result in anisotropies. Additionally, we get an energy-dependent speed when $d \neq 4$, giving dispersion.

Many of these effects are also present in the mSME, but the new operators introduce more complexity, such as direction dependence with higherorder multipoles. Dispersion results in the mSME case from $d=3$ coefficients, but it is accompanied by birefringence. The non-minimal CPT-even $c_{(I) j m}^{(d)}$ coefficients introduce the possibility of nonbirefringent dispersion. These give rise to a polarization-independent speed that depends on even powers of energy. Dispersion of this kind can be constrained by looking for arrival-time differences in explosive sources that produce photons over a high range of energies, such as GRBs.

\section{Resonant-cavity tests}

Astrophysical tests are primarily sensitive to the vacuum coefficients. Laboratory experiments complement these tests and can access the vacuumorthogonal coefficients. Many laboratory experiments rely on high-Q resonant cavities and search for tiny direction- and boost-dependent changes in frequency that would indicate Lorentz violation. ${ }^{2}$

The change in frequency can be determined perturbatively. Given the conventional electromagnetic fields $E$ and $B$ and vector potential $A_{\mu}$, the shift in resonant frequency is approximated by

$$
\frac{\delta \nu}{\nu}=-\frac{1}{4\langle U\rangle} \int d^{3} x\left(\vec{E}^{*} \cdot \delta \vec{D}-\vec{B}^{*} \cdot \delta \vec{H}\right)
$$

where

$$
\begin{aligned}
& \delta \vec{D}=\hat{\kappa}_{D E} \cdot \vec{E}+\hat{\kappa}_{D B} \cdot \vec{B}+2 \vec{k}_{A F} \times \vec{A} \\
& \delta \vec{H}=\hat{\kappa}_{H E} \cdot \vec{E}+\hat{\kappa}_{H B} \cdot \vec{B}-2\left(\hat{k}_{A F}\right)_{0} \vec{A}+2 \vec{k}_{A F} A_{0} .
\end{aligned}
$$


The $\hat{\kappa}$ matrices are combinations of $\left(\hat{k}_{F}\right)_{\kappa \lambda \mu \nu}$ operators. A problem arises when we apply this result to the nonrenormalizable case. The fields generally exhibit discontinuities at one or more surfaces of the cavity. The differential operators in Eq. (8) acting on these fields then lead to divergences. These divergences can be circumvented by smoothly extending the fields in Eq. (8) beyond the volume of the cavity. Equation (7) then gives finite shifts in resonant frequency in terms of the coefficients for Lorentz violation.

While not as sensitive as astrophysical tests are to vacuum coefficients, cavity experiments can probe the much larger set of vacuum-orthogonal coefficients. Consequently, these experiments are expected to play an important role in constraining the nonrenormalizable operators of the SME.

\section{References}

1. Data Tables for Lorentz and CPT Violation, V.A. Kostelecký and N. Russell, 2010 edition, arXiv:0801.0287v3.

2. M.A. Hohensee et al., arXiv:1006.1376; Ch. Eisele et al., Phys. Rev. Lett. 103, 090401 (2009); S. Herrmann et al., Phys. Rev. D 80, 105011 (2009); M. Mewes, Phys. Rev. D 78, 096008 (2008); H. Müller et al., Phys. Rev. Lett. 99, 050401 (2007); M. Mewes and A. Petroff, Phys. Rev. D 75, 056002 (2007); P.L. Stanwix et al., Phys. Rev. D 74, 081101 (R) (2006); S. Herrmann et al., Phys. Rev. Lett. 95, 150401 (2005); P.L. Stanwix et al., Phys. Rev. Lett. 95, 040404 (2005); P. Antonini et al., Phys. Rev. A 72, 066102 (2005); P. Wolf et al., Phys. Rev. D 70, 051902 (2004); H. Müller et al., Phys. Rev. Lett. 91, 020401 (2003); J.A. Lipa et al., Phys. Rev. Lett. 90, 060403 (2003).

3. V.A. Kostelecký and M. Mewes, Phys. Rev. D 80, 015020 (2009).

4. V.A. Kostelecký and M. Mewes, Ap. J. Lett. 689, L1 (2008).

5. V.A. Kostelecký and M. Mewes, Phys. Rev. Lett. 87, 251304 (2001); Phys. Rev. D 66, 056005 (2002); Phys. Rev. Lett. 97, 140401 (2006); Phys. Rev. Lett. 99, 011601 (2007); E.Y.S. Wu et al., Phys. Rev. Lett. 102, 161302 (2009); E. Komatsu et al., Ap. J. Suppl. 180, 330 (2009); T. Kahniashvili et al., Phys. Rev. D 78, 123006 (2008); J.-Q. Xia et al., Astron. Astrophys. 483, 715 (2008); P. Cabella et al., Phys. Rev. D 76, 123014 (2007); B. Feng et al., Phys. Rev. Lett. 96, 221302 (2006); S.M. Carroll et al., Phys. Rev. D 41, 1231 (1990).

6. J.-P. Bocquet et al., arXiv:1005.5230; B. Altschul, Phys. Rev. D 80, 091901(R) (2009); M.A. Hohensee et al., Phys. Rev. D 80, 036010 (2009); Phys. Rev. Lett. 102, 170402 (2009).

7. D. Colladay and V.A. Kostelecký, Phys. Rev. D 55, 6760 (1997); Phys. Rev. D 58, 116002 (1998). 